\title{
Regulated Expression and Function of the Somatodendritic Catecholamine Neurotransmitter Transporters
}

\author{
Shigeo Kitayama ${ }^{1, *}$ and Chiharu Sogawa ${ }^{1}$ \\ ${ }^{1}$ Department of Dental Pharmacology, Okayama University Graduate School of Medicine, Dentistry \\ and Pharmaceutical Sciences, 5-1 Shikata-cho, 2-chome, Okayama 700-8525, Japan
}

Received August 1, 2005

\begin{abstract}
Termination of neurotransmission at catecholaminergic synapses is well documented by the transporters for dopamine and norepinephrine, members of the $\mathrm{Na}^{+} / \mathrm{Cl}^{-}$-dependent neurotransmitter transporter family, which accumulates released transmitters within their nerve endings, respectively. Although somatodendritic expression of the transporters and the effects of cocaine and amphetamine on those have been reported, their role is still obscure. Recent findings of the transporter function as an ion channel and/or its reverse transport property provide a clue to identify the role of these transporters in the somatodendrites and their consequential interaction with uptake inhibitors. Differences in ionic environment and maturity of the release machinery in the somatodendrites at developmental stages influence the transporter functions, resulting in the formation of both positive and negative feedback loop of catecholaminergic neurons.
\end{abstract}

Keywords: dopamine, norepinephrine, uptake inhibitor, reverse transport, chloride current

\section{Introduction}

Catecholamine neurotransmitter transporters, such as the transporters for dopamine (DAT) and norepinephrine (NET), belong to the $\mathrm{Na}^{+} / \mathrm{Cl}^{-}$-dependent neurotransmitter transporter family and play a key role in regulating the transmitter action at synapses $(1-3)$. It is well established that these neurotransmitter transporters compose the transmitter uptake system, which serves to terminate synaptic transmission by accumulating the released neurotransmitters back into the nerve ending. Indeed, ablation of DAT and NET genes results in the prolongation of synaptic clearance of released catecholamines $(4,5)$. Therefore, as these transporters are known to be a target of psychostimulants including cocaine and amphetamines and also antidepressants $(1-3)$, these drugs have the potential to act as an enhancer of catecholaminergic neurons in the synapses of their projecting areas.

In addition, these transporters are also present in the somatodendrites, although their role is not as clear as

*Corresponding author. FAX: +81-86-235-6664

E-mail: shigeok@md.okayama-u.ac.jp

Invited article that in the axonal nerve terminals. Somatodendritic release of catecholamines has been documented, and the transporter might regulate the clearance of released transmitters $(6,7)$. Cocaine and amphetamines could thus act on DAT and NET in the somatodendrites of the dopaminergic neurons in the ventral tegmental area (VTA) or substantia nigra (SN) and noradrenergic neurons in the locus coeruleus (LC). They caused a decrease in firing rate via increase in extracellular dopamine (DA) or norepinephrine (NE) released from dendrites, which then stimulates inhibitory $\mathrm{D}_{2}$ and $\alpha_{2}$ autoreceptors $(8,9)$. Based on this scheme, the role of DAT and NET in the somatodendrites is to terminate the inhibitory action of released DA and NE by reuptake, working as an excitatory regulator of catecholaminergic neurons themselves. Therefore, the somatodendritic action of uptake inhibitors makes their effects complicated when administrated systematically.

In contrast to the classical view of the role of neurotransmitter transporters as mentioned above, there is much evidence demonstrating the transportermediated release of neurotransmitters, such as reverse transport (10). Activation of transporter reversal is expected to increase the availability of neurotransmitters at their receptors, opposite to the uptake. In somatoden- 
drites, a recent report (11) has demonstrated an example of the transporter-mediated release of neurotransmitter that the increase in reverse transport of DA by DAT through electrical stimulation of subthalamic glutamatergic neurons causes IPSP (inhibitory postsynaptic potential) via dendritic $\mathrm{D}_{2}$ receptor in $\mathrm{SN}$.

$\mathrm{Na}^{+}$-dependent net charge movement based on the experimental results indicates that the neurotransmitter transporter is electrogenic. In addition, there is a line of evidence suggesting that the neurotransmitter transporters act as ion channels (12). Recently, Ingram et al. (13) suggested that the DA-induced inward currents associated with DAT modulate the excitability of midbrain DA neurons and may regulate DA release. More recently, Carvelli et al. (14) reported that DAT not only transports DA but also exhibits a channel mode of conduction that directly modulates membrane potential and neuronal function in C.elegans. These results suggest that tonic activity excites these neurons by activating an uncoupled $\mathrm{Cl}^{-}$conductance that is mediated by DAT.

Consequently, cocaine and amphetamine may exert different effects on neuron excitability via DAT-associated current. If DAT-associated current depolarizes the somatodendrite of a DA neuron, cocaine produces hyperpolarization by preventing DA action on DAT without affecting extracellular DA concentration. On the contrary, amphetamine, a transportable substrate for DAT, may elicit DAT-associated current by itself, resulting in depolarization directly as well as DA (15).

Differential properties of the somatodendritic transporters may be due not only to the transporters themselves, but also to the environments that influence the expression and function of the transporters. Therefore, overall effect of those drugs depends on how many transporters the somatodendrites (SN/VTA and LC) express in relation to the projecting areas (for example, the striatum and the nucleus accumbens for the dopaminergic pathway) and how well the somatodendritic transporters work in comparison with the transporters expressed in nerve endings in the projecting areas. Chen and Reith (16) reviewed the role of somatodendritic monoamine transporters in line with the action of monoamine uptake blockers in the first edition of the book entitled "Neurotransmitter Transporters: Structure, Function, and Regulation". Despite their efforts to clarify the role of somatodendritic transporters, there are still unresolved questions, so far.

In this article, we addressed these issues and reviewed the recent findings of the regulated expression and function of the somatodendritic catecholamine transporters in the central nervous system (CNS).

\section{Regulated expression in somatodendrite}

Expression of the catecholamine transporters in the brain regions was examined in vitro by autoradiography and immunohistochemistry and in vivo by PET and SPECT. There is a line of evidence demonstrating that DAT expression is relatively poor in VTA and SN compared to that in the striatum or nucleus accumbens, while NET is most abundant in LC (17). A recent study reported that during development in the rat, DAT density increased from E18 to P18 where it reaches the adult level and then remains stable until P70 (18). In contrast, NET development in the brainstem was found to exhibit elevated densities at P5, 10, and 20 that decreased in the adult. LC, in paticular, had very high NET expression in the early postnatal period that decreased dramatically in the adult brain (19). From these results, questions arise as to whether systemic application of cocaine exerts different effects on dopaminergic and adrenergic systems through somatodendritic DAT and NET and whether its effect alters during development.

It is conceivable that systemic application of cocaine primarily causes a decrease in cell firing as a consequence of the increase in extracellular $\mathrm{NE}$ and its stimulation of inhibitory $\alpha 2$ autoreceptor in the somatodendritic regions of $\mathrm{LC}$, which results in the decreasing effects on the projecting areas. In contrast, the dominant change in DA neurons after systemic treatment with cocaine should be an increase in DA at nerve endings in the projecting area with little change of firing rate due to a least expression of DAT in the somatodendritic regions of VTA and SN.

Although mechanisms underlying the differences in somatodendritic expression between DAT and NET remain to be established, there are several clues to clarify their expression. Heterologous expression of monoamine transporters in a polarized cell line derived from Madin-Darby canine kidney (MDCK) demonstrated that DAT was located primarily on the apical surface, while NET was found on the basolateral membranes (20). These results are consistent with the differences in the somatodendritic distribution of these transporters mentioned above. Although NET and DAT share more than $60 \%$ amino acids in their structure, they are localized on opposite poles of MDCK cells. Therefore, there may be significant determinants that participate in the different expression.

The dileucine motif is a basolateral trafficking signal present in the N-terminus of NET but not in DAT. Recently, Gu et al. (21) demonstrated that the aminoterminus of NET contains a basolateral localization signal for epithelial cells. However, substitution of dileucine in NET did not disrupt basolateral localization, 
indicating the presence of additional signals in NET directing its basolateral localization. Another candidate motif is $\operatorname{Yxx} \Phi$ present in the C-terminus of NET. However, DAT also contains this motif.

There may be a difference in the associated proteins that regulate trafficking and internalization of the transporters. Recent observation indicated PICK1 as the interacting subcellular protein with DAT and also with NET at their C-terminal PDZ binding domain (22). It seems likely to assume that a series of interacting proteins may specifically modulate DAT and NET, respectively.

Less expression in somatodendrites occurs not only due to the directed trafficking to the nerve ending but also by the accelerated turnover in the somatodendrites. Kimmel et al. (23) reported the synthesis rate, degradation rate constant, and half-life of DAT in the rat striatum and nucleus accumbens after administration of the irreversible DAT ligand RTI-76 [3 $\beta$-(3- $p$-chlorophenyl)tropan- $2 \beta$-carboxylic acid $p$-isothiocyanatophenylethyl ester hydrochloride]. They demonstrated the turnover of DAT in the nerve ending (striatum and nucleus accumbens), but it is uncertain in the somatodendrites (VTA and SN).

If turnover rate of the transporter in the somatodendrites is higher than that in the nerve ending, cell surface expression in the somatodendrites is apparently less. This may occur as a consequence of accelerated internalization and degradation. Daniels and Amara (24) demonstrated that the human DAT at the cell surface of MDCK cells was rapidly endocytosed into early endosomes and completely degradated after PKC activation, although recycling of internalized DAT was reported in PC12 cells (25). The different fate of internalized DAT may be due to the different cell lines used or use of GFP-tagged DAT or untagged DAT. Alternatively, the intrinsic cellular mechanism underlying DAT recycling was involved in PC12 cells that possess native NET. Nonclassical, distinct endocytic signals were newly identified in neurotransmitter transporters including both DAT and NET (26); thus, this seems unlikely to participate in their differential expression.

\section{Excitability regulated by the reverse transport}

It has been established that neurotransmitter transporters transport substrates bidirectionally, although mechanisms that trigger the reverse transporter are not clear. It is believed that since a $\mathrm{Na}^{+}$gradient provides a driving force for the forward transport (uptake), intracellular accumulation of $\mathrm{Na}^{+}$, and consequently decrease in or reversal of $\mathrm{Na}^{+}$gradient across the plasma membrane suppress neurotransmitter uptake, and occa- sionally brings about reverse transport. There is evidence indicating the reverse transport in such examples observed in physiological and pathophysiological conditions (10).

Anoxia and ischemia induce catecholamine release probably by both exocytosis and reverse transport. ATP depletion induced by hypoxia and hypoglycemia causes inhibition of $\mathrm{Na}^{+}, \mathrm{K}^{+}$-ATPase, resulting in the accumulation of intracellular $\mathrm{Na}^{+}$. Intracellular acidification at that time activates $\mathrm{Na}^{+}, \mathrm{H}^{+}$antiporter, so that it facilitates $\mathrm{Na}^{+}$accumulation (27). Is there any route for $\mathrm{Na}^{+}$influx under physiological conditions? In somatodendrites, a part of many inputs makes synapses where receptoroperated $\mathrm{Na}^{+}$channels synergically act to excite the plasma membrane. Consequent accumulation of intracellular $\mathrm{Na}^{+}$causes neurotransmitter release through the transporter by its reversal. As mentioned in the Introduction, Falkenburger et al. (11) reported that the reverse transport likely occurs under physiological conditions, but may depend not only on intracellular accumulation of $\mathrm{Na}^{+}$by ionotropic glutamate receptors but also on something other than intracellular $\mathrm{Na}^{+}$by metabotropic glutamate receptors.

Facilitated exchange diffusion theory (28) has been proposed for the release of catecholamines by weak basic amines including amphetamine. Since amphetamine is a substrate for the monoamine transporters, uptake of amphetamine produces the accumulation of inward-facing conformations of the transporters, and these bind substrate and extrude it, although a question arises as to why inward-facing transporter preferentially binds and extrudes the neurotransmitter rather than amphetamine (e.g., Ref. 29 as evidence inconsistent with the theory). The recent study by Seidel et al. (30) addressed this issue in view of the oligomer-based counter-transport model: influx and efflux occur through separate but coupled moieties and both $\mathrm{Na}^{+}$accumulation and $\mathrm{PKC}$-dependent phosphorylation resulting from amphetamine uptake are required. The authors proposed that the latter action is the crucial switch between concomitant and sequential counter-transport. The finding that removal of amino terminal PKCconsensus phosphorylation sites abolishes the amphetamine-induced transport reversal in DAT (31) supports this idea. According to this, the mechanism underlying the DAT-mediated release of DA from somatodendrites of dopaminergic neuron in $\mathrm{SN}$ as mentioned above (11) could be explained by the DAT phosphorylation induced by metabotropic glutamate receptor activation, as predicted by Blakely (32).

Are there any factors in favor of the reverse transport in somatodendrites? Sufficient increase in cytosolic substrates is prerequisite to the reverse transport. It may 
depend on the possible cytosolic pool of substrates, which associates closely with the transporter, especially in its outward direction, and responds to a driving signal for reverse transport. Studies using electron microscopic immunohistochemistry demonstrated the specialized architecture of DAT expression for transmitter release machinery in the somatodendritic region of dopaminergic neurons, showing the co-existence of VMAT localized mainly on smooth endoplasmic reticulum membrane and DAT on the plasma membrane in tyrosine hydroxylase-positive dendrites and perikarya $(33-35)$. Less available vesicles for exocytotic release, such as dense core vesicles or small synaptic vesicles, were observed there. Transmitter synthesis activity is considered to have an important correlation with the reverse transport. Another factor that provides the cytosolic pool of transmitter for reverse transport is a vesicular release (leakage) of substrates into cytosol. It has been proposed that intracellular accumulation of $\mathrm{Na}^{+}$could be responsible for the increase in net leakage of NE from the storage vesicles (36). Taken together, however, there is no direct evidence indicating that it occurs favorably in somatodendrites.

What is the consequence of the inhibition of transporter-mediated release by an uptake blocker such as cocaine in somatodendrites? In DA neurons in the SN, somatodendritic release of DA stimulates $\mathrm{D}_{2}$ DA receptors on the same cell or neighboring cells to inhibit cell firing rate, resulting in feedback inhibition and inhibitory volume transmission. By blocking the reverse transport, the inhibitor should cause enhancement rather than inhibition of cell excitability. However, this conclusion is not consistent with observations that cocaine causes a decrease in firing rate in VTA and SN, as mentioned in the Introduction $(8,9)$. The discrepancy may be resolved by the recent findings that afferent modulation of DA neuron firing rate differentially regulates tonic and phasic DA transmission (37).

\section{Excitability regulated by the channel mode action}

It is conceivable that both transport-associated current and transport-independent (channel mode) current participate in the changes in the plasma membrane potential. Important issue to be resolved is as to whether DAT/NET current causes depolarization or hyperpolarization. The presently available data including the reports mentioned in the Introduction $(13,14)$ all indicate that the transporter-mediated current causes depolarization. However, those data strongly suggest that the charge carrier is $\mathrm{Cl}^{-}$in their channel mode. This suggests that transporter-mediated current depends at least in part on the $\mathrm{Cl}^{-}$equilibrium potential determined by the electrochemical gradient of $\mathrm{Cl}^{-}$across the plasma membrane. This lead to the assumption that chlorideregulatory mechanisms play an important role in regulating the transporter-mediated current and that the intrinsic property of those in somatodendrites or nerve endings of catecholaminergic neurons may differently affect cell excitability.

There are at least four $\mathrm{Cl}^{-}$permeation pathways across the plasma membrane known to regulate the intracellular $\mathrm{Cl}^{-}$concentration in the CNS: the $\mathrm{Na}^{+}-\mathrm{K}^{+}-2 \mathrm{Cl}^{-}$cotransporter $\mathrm{NKCC} 1$, the $\mathrm{K}^{+}-\mathrm{Cl}^{-}$co-transporter $\mathrm{KCC} 2$, the voltage-gated chloride channel $\mathrm{ClC} 2$, and the sodium-dependent anion $\left(\mathrm{Cl}^{-}-\mathrm{HCO}_{3}^{-}\right)$exchanger SDAE (38). Developmental changes in the expression of these proteins are considered to underlie the excitatory actions of GABA during development (39). It has been shown that neuron-specific $\mathrm{KCC} 2$ efficiently extrude $\mathrm{Cl}^{-}$to generate an inwardly directed $\mathrm{Cl}^{-}$electrochemical gradient (38). Under this condition, opening of a $\mathrm{Cl}^{-}$ channel, for example, $\mathrm{GABA}_{\mathrm{A}}$ receptor, causes hyperpolarization, resulting in the inhibition of cell excitability. On the other hand, inward-directing $\mathrm{Cl}^{-}$transport by NKCC1 shifts the $\mathrm{Cl}^{-}$equilibrium potential to depolarization. Recent work has shown that SDAE and $\mathrm{ClC} 2$ are likely to serve for maintaining the low intracellular $\mathrm{Cl}^{-}$concentration in nigral dopaminergic neurons, and the relatively low efficacy of $\mathrm{GABA}_{\mathrm{A}}$ receptor-mediated inhibition in these neurons may be related to their lack of KCC2 (40). Interestingly, they also showed that estimates for intracellular $\mathrm{Cl}^{-}$concentration of dopaminergic neuron yield values of 8 and $17 \mathrm{mM}$ in the presence and absence of bicarbonate, indicating a profound bicarbonate dependence of $\mathrm{Cl}^{-}$extrusion in dopaminergic neurons. Since intracellular bicarbonate tightly couples with $\mathrm{H}^{+}$for regulating intracellular $\mathrm{pH}$, influx of $\mathrm{Na}^{+}$, which is associated always with substrate transport by the transporter, must modify $\mathrm{Cl}^{-}$ equilibrium. This may explain the difference between $\mathrm{GABA}_{\mathrm{A}}$-mediated and DAT-mediated current observed by Ingram et al. (13).

The physiological significance of the transportermediated current is still unknown. The results of Ingram et al. (13) clearly demonstrated that low levels of extracellular DA increase excitability of midbrain DA neurons in culture via DAT-mediated conductances, while DA at higher levels suppresses it through $\mathrm{D}_{2}$ autoreceptor, suggesting that this could occur in somatodendrites of DA neurons. However, it should be noted that this depends on the chloride-regulatory mechanisms mentioned above. Physiological relevance of NETmediated current in the somatodendrite of LC adrenergic neurons remains to be elucidated. 


\section{Methylphenidate, amphetamine, and ADHD}

Somatodendritic expression of catecholamine transporters is important issue to consider for the pathogenesis of attention-deficit hyperactivity disorder (ADHD) since there is a line of evidence demonstrating that changes in dopaminergic and adrenergic activity with DAT and perhaps NET in CNS are involved in ADHD (41). Methylphenidate and amphetamine are used to treat a large number of children with ADHD. How do these stimulants reduce hyperactivity?

Seeman and Madras (42) described a hypothesis to answer this apparent discrepancy of the action of stimulants; methylphenidate at low doses elevates resting DA, which activates the presynaptic $\mathrm{D}_{2-}$ inhibitory autoreceptor to lower the impulse-triggered release of DA. However, it has been shown that burst firing induces massive synaptic DA release, which is rapidly removed by reuptake before escaping the synaptic cleft, whereas increased population activity modulates tonic extrasynaptic DA levels that are less influenced by reuptake (37). Therefore, it is reasonable to consider the action of stimulants on somatodendrites to reduce the phasic activity of DA neurons, as mentioned in section 2. However, this idea is inconsistent with the recent finding from the examination with PET using the selective radioligand $\left[{ }^{11} \mathrm{C}\right] \mathrm{PE} 21$ indexing DAT that midbrain DAT binding is decreased in male adolescents with ADHD (43). The alteration may reflect compensatory, rather than causal phenomena. Further study is needed to clarify this.

DAT-mediated change in conductances in midbrain DA neurons (13) may contribute mainly to tonic activity, but not phasic activity, of DA neurons, resulting in less participation in ADHD symptoms. However, in the early stage of development, DAT/NET may cause depolarization in the somatodendrites, if $\mathrm{Cl}^{-}$equilibrium potential does not shift sufficiently to the hyperpolarizing potential. Under this condition, DAT/NET in somatodendrites works as an excitatory regulator of DA/NE neuron activity in the early stage of development. An uptake inhibitor such as methylphenidate may suppress this to operate its activity in ADHD treatment, although amphetamine must exert the opposite effect according to this hypothesis. Taken together, important issues to clarify the pathogenesis of ADHD in relation to DAT/NET include not only the expressional changes of DAT/NET between somatodendrites and nerve endings but also the environmental changes that influence DAT/NET functions.

\section{Dopaminergic nerve ending}

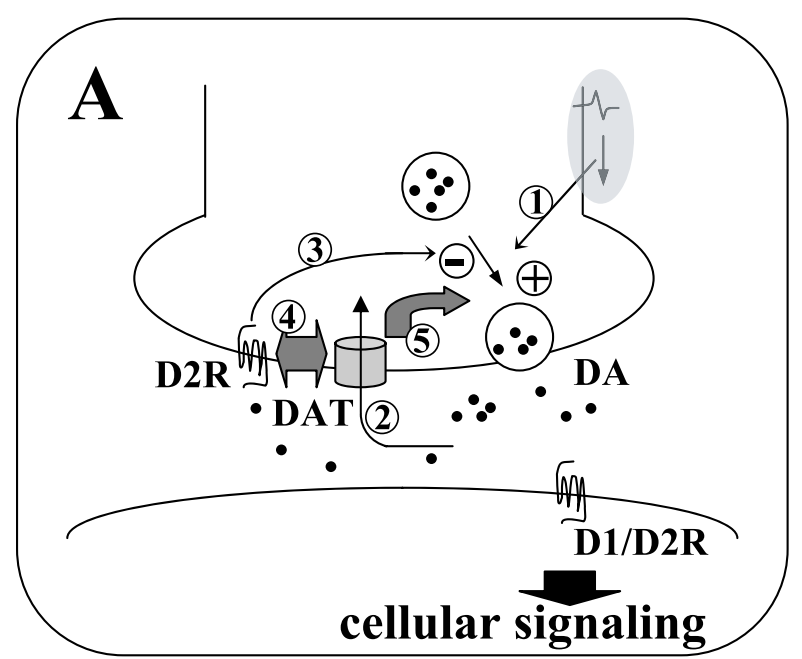

Dopaminergic dendrite

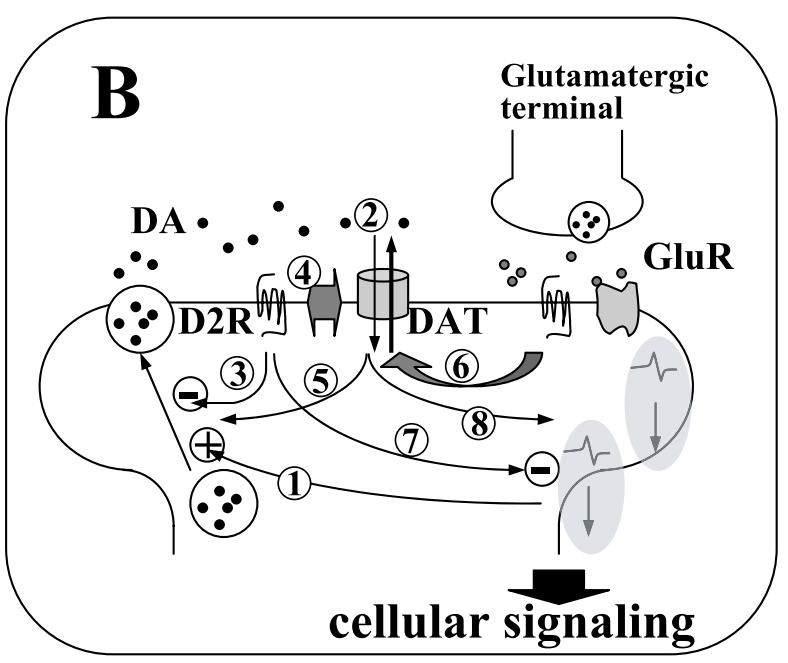

Fig. 1. Illustration of the nerve terminal (A) and somatodendritic (B) dopamine transporter (DAT) that differentially participates in the regulation of synaptic or extrasynaptic neurotransmission. A: Excitation of nerve ending causes an exocytotic release of dopamine (DA, 1), followed by reuptake by DAT (2) in coordination with the release inhibition by dopamine $\mathrm{D}_{2}$ receptor (D2R, 3). D2R regulates the expression and function of DAT (4), and DAT-mediated regulation of extracellular DA concentration also affects excitability of nerve ending through D2R (4). Furthermore, electrical activity of DAT directly modulates excitability of nerve ending to participate in exocytosis (5). Possibility of DAT reversal in nerve ending is not included in this panel. B: Similar processes occur in dendrite $(1-5)$, with some differences as described in the text. Activation of metabotropic glutamate receptor (GluR) causes DA release through the reversal of DAT (6), followed by the inhibition of firing rate through D2R (7), in response to burst firing (phasic state). Action of DAT in the channel mode directly affects excitability (8), especially at low extracellular DA concentrations corresponding to the tonic state of dendritic activity. See text for details. 


\section{Concluding remarks}

Several different features of the role of transporters between nerve endings and somatodendrites are summarized in Fig. 1. Recent findings of the transporter function as ion channel and/or reverse transport property provide a clue to identify their role in the somatodendrites and the consequential interaction with uptake inhibitors. Differences in ionic environment and maturity of release machinery in the somatodendrites at developmental stages influence the transporter functions, resulting in the formation of both a positive and a negative feedback loop of catecholaminergic neurons. Drawing a complete picture for specialized transporter function and expression in somatodendrites remains in progress.

\section{References}

1 Iversen LL. Role of transmitter uptake mechanisms in synaptic neurotransmission. Br J Pharmacol. 1971;41:571-591.

2 Amara SG, Kuhar MJ. Neurotransmitter transporters: recent progress. Ann Rev Neurosci. 1993;16:73-93.

3 Kitayama S, Dohi T. Cellular and molecular aspects of monoamine neurotransmitter transporters. Jpn J Pharmacol. 1996;72: 195-208.

4 Giros B, Jaber M, Jones SR, Wightman RM, Caron MG. Hyperlocomotion and indifference to cocaine and amphetamine in mice lacking the dopamine transporter. Nature. 1996; 379:606-612.

$5 \mathrm{Xu} \mathrm{F}$, Gainetdinov RR, Wetsel WC, Jones SR, Bohn LM, Miller $\mathrm{GW}$, et al. Mice lacking the norepinephrine transporter are supersensitive to psychostimulants. Nature Neurosci. 2000;3: 465-471.

6 Ludwig M, Pittman QJ. Talking back:dendritic neurotransmitter release. Trends Neurosci. 2003;26:255-261.

7 Cragg SJ, Rice ME. DAncing past the DAT at a DA synapse. Trends Neurosci. 2004;27:270-277.

8 Bunney BS, Aghajanian GK, Roth RH. Comparison of effects of L-DOPA, amphetamine and apomorphine on firing rate of rat dopaminergic neurons. Nature. 1973;245:123-125.

9 Svensson TH, Usdin T. Feedback inhibition of brain noradrenaline neurons by tricyclic antidepressants: $\alpha$-receptor mediation. Science. 1978;202:1089-1091.

10 Levi G, Raiteri M. Carrier-mediated release of neurotransmitters. Trends Neurosci. 1993;16:415-419.

11 Falkenburger BH, Barstow KL, Mintz IM. Dendrodendritic inhibition through reversal of dopamine transport. Science. 2001;293:2465-2470.

12 Sonders MS, Amara SG. Channels in transporters. Curr Opin Neurobiol. 1996;6:294-302.

13 Ingram SL, Prasad BM, Amara SG. Dopamine transportermediated conductances increase excitability of midbrain dopamine neurons. Nature Neurosci. 2002;5:971-978.

14 Carvelli L, McDonald PW, Blakely RD, DeFelice LJ. Dopamine transporters depolarize neurons by a channel mechanism. Proc Natl Acad Sci U S A. 2004;101:16046-16051.
15 Sulzer D, Galli A. Dopamine transport currents are promoted from curiosity to physiology. Trends Neurosci. 2003;26:173176.

16 Chen NH, Reith MEA. Role of Axonal and somatodendritic monoamine transporters in action of uptake blockers. In: Reith MEA, editor. Neurotransmitter transporters: structure, function, and regulation. 1st ed. Totowa, NJ: Humana Press Inc; 1997. p. 345-391.

17 Javitch JA, Strittmatter SM, Snyder SH. Differential visualization of dopamine and norepinephrine uptake sites in rat brain using $\left[{ }^{3} \mathrm{H}\right]$ mazindol autoradiography. J Neurosci. 1985;5:15131521 .

18 Galineau L, Kodas E, Guilloteau D, Vilar MP, Chalon S. Ontogeny of the dopamine and serotonin transporters in the rat brain: an autoradiographic study. Neurosci Lett. 2004;363:266271.

19 Sanders JD, Happe HK, Bylund DB, Murrin LC. Development of the norepinephrine transporter in the rat CNS. Neuroscience. 2005;130:107-117.

$20 \mathrm{Gu} H \mathrm{H}$, Ahn J, Caplan MJ, Blakely RD, Levey AI, Rudnick G. Cell-specific sorting of biogenic amine transporters expressed in epithelial cells. J Biol Chem. 1996;271:18100-18106.

$21 \mathrm{Gu} \mathrm{HH}, \mathrm{Wu}$ X, Giros B, Caron MG, Caplan MJ, Rudnick G. The NH2-terminus of norepinephrine transporter contains a basolateral localization signal for epithelial cells. Mol Biol Cell. 2001;12:3797-3807.

22 Torres G, Yao WD, Mohn AR, Quan H, Kim KM, Levey AI, et al. Functional interaction between monoamine plasma membrane transporters and the synaptic PDZ domain-containing protein PICK1. Neuron. 2001;30:121-134.

23 Kimmel HL, Carroll FI, Kuhar MJ. Dopamine transporter synthesis and degradation rate in rat striatum and nucleus accumbens using RTI-76. Neuropharmacology. 2000;39:578585.

24 Daniels GM, Amara SG. Regulated trafficking of the human dopamine transporter. Clathrin-mediated internalization and lysosomal degradation in response to phorbol esters. J Biol Chem. 1999;274:35794-35801.

25 Melikian, Buckley KM. Membrane trafficking regulates the activity of the human dopamine transporter. J Neurosci. 1999; 15:7699-7710.

26 Holton KL, Loder MK, Melikian HE. Nonclassical, distinct endocytic signals dictate constitutive and PKC-regulated neurotransmitter transporter internalization. Natue Neurosci. 2005;7:881-888.

27 Shomig A, Kurz T, Richardt G, Schomig E. Neuronal sodium homeostasis and axoplasmic amine concentration determine calcium-independent noradrenaline release in normoxic and ischemic rat heart. Circ Res. 1988;63:214-226.

28 Fischer JF, Cho AK. Chemical release of dopamine from striatal homogenates: evidence for an exchange diffusion model. J Pharmacol Exp Ther. 1979;208:203-209.

29 Sitte HH, Hiptmair B, Zwach J, Pifl C, Singer EA, Scholze P. Quantitative analysis of inward and outward transport rates in cells stably expressing the cloned human serotonin transporter: inconsistencies with the hypothesis of facilitated exchange diffusion. Mol Pharmacol. 2001;59:1129-1137.

30 Siedel S, Singer EA, Just H, Farhan H, Scholze P, Kudlacek O, et al. Amphetamines take two to tango: an oligomer-based counter-transport model of neurotransmitter transport explores 
the amphetamine action. Mol Pharmacol. 2005;67:140-151.

31 Khoshbouei H, Sen N, Guptaroy B, Johnson L, Lund D, Gnegy $\mathrm{ME}$, et al. N-terminal phosphorylation of the dopamine transporter is required for amphetamine-induced efflux. PLoS Biol. 2004;2:387-393.

32 Blakely RD. Dopamine's reversal of fortune. Science. 2001;293:2407-2409.

33 Nirenberg MJ, Vaughan RA, Uhl GR, Kuhar MJ, Pickel VM. The dopamine transporter is localized to dendritic and axonal plasma membranes of nigrostriatal dopaminergic neurons. J Neurosci. 1996;16:436-447.

34 Nirenberg MJ, Chan J, Liu Y, Edwards RH, Pickel VM. Ultrastructural localization of the vesicular monoamine transporter-2 in midbrain dopaminergic neurons: potential sites for somatodendritic storage and release of dopamine. J Neurosci. 1996;16:4135-4145.

35 Nirenberg MJ, Chan J, Vaughan RA, Uhl GR, Kuhar MJ, Pickel VM. Immunogold localization of the dopamine transporter: an ultrastructural study of the rat ventral tegmental area. J Neurosci. 1997; 17:5255-5262.

36 Stite N, Trendelenburg U. The outward transport of axoplasmic noradrenaline induced by a rise of the sodium concentration in the adrenergic nerve endings of the rat vas deferens. Naunyn Schmiedebergs Arch Pharmacol. 1984;327:124-132.
37 Floresco SB, West AR, Ash B, Moore H, Grace AA. Afferent modulation of dopamine neuron firing differentially regulates tonic and phasic dopamine transmission. Nature Neurosci. 2003;6:968-973.

38 Payne JA, Rivera C, Voipio J, Kaila K. Cation-chloride cotransporters in neuronal communication, development and trauma. Trends Neurosci. 2003;26:199-206.

39 Ben-Ari Y. Excitatory actions of GABA during development: the nature of the nurture. Nature Rev Neurosci. 2002;3:728-739.

40 Gulacsi A, Lee CR, Sik A, Viitanen T, Kaila K, Tepper JM, et al. Cell type-specific differences in chloride-regulatory mechanisms and GABAA receptor-mediated inhibition in rat substantia nigra. J Neurosci. 2003;23:8237-8246.

41 Sagvolden T, Sergeant JA. Attention deficit/hyperactivity disorder-from brain dysfunctions to behaviour. Behav Brain Res. 1998;94:1-10.

42 Seeman P, Madras B. Methylphenidate elevates resting dopamine which lowers the impulse-triggered release of dopamine: a hypothesis. Behav Brain Res. 2002;130:79-83.

43 Jucaite A, Fernell E, Halldin C, Forssberg H, Farde L. Reduced midbrain dopamine transporter binding in male adolescents with attention-deficit/hyperactivity disorder: association between striatal dopamine markers and motor hyperactivity. Biol Psychiatry. 2005;57:229-238. 